\title{
Interacciones de metadona con fármacos antiinfecciosos y sustancias de abuso
}

\author{
Moreno Brea, M.R. * Rojas Corrales, M.O.**; Gibert-Rahola, J.***; Mico J.A.**** \\ * Dpto. Neurociencias. Universidad de Cádiz. E.U. Ciencias de la Salud. Prof. Asociada. \\ ** Dpto. Neurociencias. Universidad de Cádiz. Facultad de Medicina. Becaria F.P.I. \\ *** Dpto. Neurociencias. Universidad de Cádiz. Facultad de Medicina. Catedrático de Farmacología. \\ ** Dpto. Neurociencias. Universidad de Cádiz. Facultad de Medicina. Prof. Titular de Farmacología. \\ Enviar correspondencia a: \\ Prof. Dr. Juan Antonio Micó Segura. Dpto. Neurociencias. Facultad de Medicina. Pza. Fragela S/N. 11003 Cádiz. Teléfono 956228717.
}

\section{Resumen:}

Frecuentemente es necesario utilizar antiinfecciosos en pacientes tratados con metadona. Se han observado diversas interacciones entre metadona y fármacos antiinfecciosos, siendo necesaria una especial vigilancia en caso de tratamiento antirretroviral. Se ha descrito aumento de los niveles plasmáticos y de la toxicidad de zidovudina por metadona; también es posible el efecto contrario, es decir la disminución de los efectos del opiáceo por la zidovudina. Entre los inhibidores de la proteasa, ritonavir puede aumentar considerablemente los niveles plasmáticos de metadona y sus efectos depresores. Eritromicina o ketoconazol tienen efectos farmacocinéticos similares. Al contrario, se ha observado el desencadenamiento de síndrome de abstinencia debido a inducción metabólica originada por rifampicina. También las sustancias de abuso, frecuentemente utilizadas por estos pacientes, pueden originar interacciones farmacológicas. La significación clínica de la interacción alcohol/metadona no está clara, en ella pueden participar modificaciones en el metabolismo hepático. A pesar del extenso abuso de cocaína en estos pacientes, son poco conocidas las consecuencias de su consumo con metadona, en las que pudieran estar implicadas interacciones entre la neurotransmisión catecolaminérgica y opioidergica. Se requieren más estudios sobre cannabinoides, anfetaminas o drogas de diseño, de frecuente consumo en esta población.

Palabras clave: Metadona. Interacciones farmacológicas. Drogas de abuso. Alcohol. Antiinfecciosos. Nicotina.

\section{Summary:}

Antiinfection therapy is frequently needed in patients undergoing methadone treatment. Interactions between methadone and antiinfectious drugs occur and antirretroviral treatment must be closely monitored. It is known that zidovudine levels and hence toxicity may be increased by methadone; conversely, xidovudine may decrease the opiate effect. Among the protease inhibitors, ritonavir is likely considerably to increase plasma levels of methadone and its depressive effects. Erythromycin or ketoconazole may also raise methadone plasma concentrations. On the other hand, methadone withdrawal symptoms have been reported in addicts treated with the tuberculostatic rifampicin, due to the induction of the drug metabolism. Different types of abuse substances are also commonly used in opiate-dependent patients. These substances may also cause interactions with methadone. The clinical significance of alcohol and methadone combinaton remains uncertain, although alterations in hepatic metabolism may be implicated. Abuse of cocaine in methadone-maintained patients has been extensively reported. The consequences of cocaine consumption on maintenance treatments are not clear, although catecholaminergic modulation of opioidergic neurotransmission could play and important role. Although they are also widely consumed by patients on methadone therapy, little is known about the final consequences of cannabinoids, amphetamines or novel "designer drugs" on methadone treatments.

Key words: Methadone. Drugs-interactions. Abuse substances. Alcohol. Antiinfectious drugs. Nicotine.

\section{INTRODUCCIÓN}

$\mathbf{L}$ a modificación cualitativa o cuantitativa de los efectos de un fármaco como consecuencia de la presencia o acción simultánea de otro u otros fármacos, es decir, la aparición de interacciones medicamentosas, es una posibilidad a considerar y vigilar siempre que se administran varias sustancias medica- mentosas a un mismo sujeto. El riesgo de aparición de interacciones se incrementa, evidentemente, con el número de principios activos coadministrados. Con frecuencia las consecuencias de las mismas no sólo no son beneficiosas sino que pueden resultar incluso nocivas.

Los pacientes incluidos en programas de mantenimiento o deshabituación a opiáceos (en concreto, con 
metadona, el agonista opiáceo más utilizado en nuestro medio) deben constituir objeto de un especial interés en la vigilancia de las interacciones medicamentosas, pues sus consecuencias pueden ser potencialmente peligrosas e incluso modificar la adhesión al programa sustitutivo. Además, en ellos existe, sin duda, una mayor posibilidad de presentación de interacciones medicamentosas dada la elevada probabilidad de confluencia de diversos factores interrelacionados (Tabla I). Entre estos destacan la utilización controlada (bajo prescripción) de medicamentos para el tratamiento de diversas patologías (con particular importancia de los cuadros infecciosos asociados a VIH y de trastornos psiquiátricos), así como la frecuente y considerable ingesta de otras sustancias de abuso dentro de un cua- dro de politoxicomanía, que pueden modificar el efecto neto de los opiáceos. Existe, en fin, la posibilidad no desestimable, de que coexistan alteraciones, orgánicas y/o funcionales, de los órganos encargados de la eliminación de los fármacos del organismo, sobre todo del hígado, órgano biotransformante por excelencia, pero también del riñón. Como consecuencia, se vería modificada la permanencia de los fármacos en el organismo y los niveles plasmáticos y tisulares alcanzados. Tales alteraciones pueden derivar de la propia historia toxicológica (efectos heptotóxicos de sustancias de abuso y adulterantes) o de otras circunstancias asociadas a la misma (hepatopatías crónicas por infección vírica -destacando hepatitis B y $\mathrm{C}-$, cirrosis,...).

\section{Tabla I. Causas de la mayor susceptibilidad de los pacientes en tratamiento con metadona}

a las interacciones medicamentosas.

POLIMEDICACIÓN:

-Tratamiento patología infecciosa asociada (VIH, TBC, VHB, VHC, ...).

- Politerapia

- Terapia agresiva

- Fármacos tóxicos

- Alteraciones orgánicas múltiples

- Alteraciones funcionales debidas al trastorno inmunológico

-Tratamiento patología psiquiátrica asociada: uso de benzodiacepinas, antidepresivos, antipsicóticos,...

UTILIZACIÓN DE PSICOFÁRMACOS COMO SUSTANCIAS DE ABUSO

EMPLEO DE SUSTANCIAS DE ABUSO NO MEDICAMENTOSAS:

Alcohol, Anfetaminas, Cánnabis, Cocaína, Tabaco,...

LESIONES DE LOS ÓRGANOS ENCARGADOS DE LA ELIMINACIÓN DE LOS FÁRMACOS:

Hígado, Riñón (historia toxicológica y patológica)

Parece, pues, a todas luces necesario suscitar una especial sensibilidad y atención ante la posibilidad de interacciones y efectos adversos medicamentosos en los individuos incluidos en programas de mantenimiento o deshabituación a opiáceos. No obstante, ésta es una labor muy complicada, ante el desconocimiento del terapeuta sobre la naturaleza y cantidad exacta de las sustancias ingeridas por el paciente. A veces sólo puede sospecharse ante el descubrimiento en estos pacientes de efectos inesperados, por su magnitud o tipo, de los medicamentos que reciben. A continuación se revisan las interacciones más documentadas de la metadona (el opiáceo más empleado en estos programas) con fármacos antiinfecciosos y con sustancias de abuso no medicamentosas.

\section{Características farmacocinéticas de la metadona}

Aunque con frecuencia se pueden observar modificaciones de los efectos farmacológicos de la metado- na como consecuencia de la presencia de otras sustancias (interacciones de carácter farmacodinámico, en forma, sobre todo, de adición de efectos depresores centrales), también son posibles interacciones de índole farmacocinético, es decir, que originan cambios en las concentraciones plasmáticas de alguno de los fármacos implicados en ellas. Pueden ser el resultado de interferencias en uno o más de los procesos que condicionan tales niveles (absorción, distribución, metabolización o excreción). Cuando como consecuencia de la interacción se ocasiona un aumento de los niveles plasmáticos del opiáceo, pueden originarse un incremento de sus efectos reforzadores, pero también de algunos de sus efectos adversos dosis-dependientes (destacando por su gravedad la depresión del SNC). Resulta, pues, conveniente resaltar los aspectos más relevantes de la farmacocinética de la metadona.

La metadona (figura 1) es un agonista opiáceo puro ligeramente más potente que la morfina con la que comparte sus propiedades farmacológicas. Por su 
Figura 1. Estructura química de la metadona

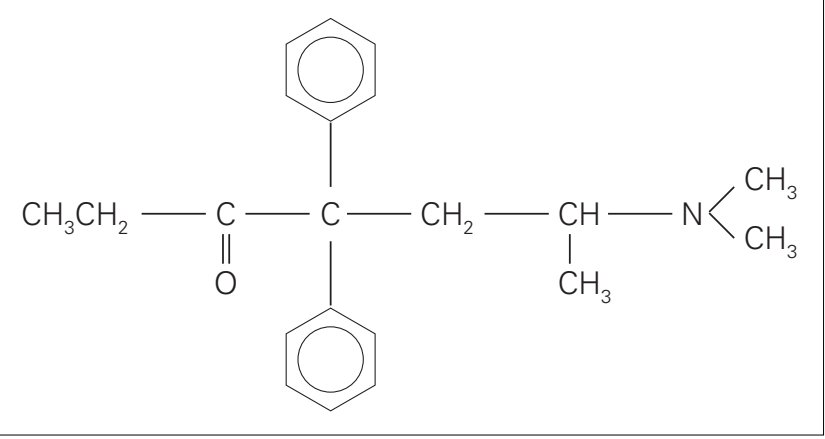

carácter agonista, produce tolerancia y dependencia física y psicológica. Presenta muy buena absorción oral, apareciendo en plasma a la media hora de la ingestión; alcanza concentraciones máximas a las cuatro horas aproximadamente de la administración (2-4 h.). Las concentraciones máximas cerebrales se alcanzan en el plazo de 1-2 horas tras administración parenteral (s.c. o i.m.) correlacionándose bien con los efectos analgésicos.

Se une en elevada proporción a las proteínas plasmáticas (90\%), por lo cual variaciones en la cantidad de proteínas (posibles en hepatopatías severas) modificarían la concentración de metadona libre. Se fija con intensidad a proteínas tisulares. Su administración repetida provoca una acumulación gradual en los tejidos. Esta fracción funciona como órgano de reserva, de forma que, al interrumpirse su administración, se conservan las concentraciones plasmáticas bajas por liberación lenta desde los sitios extravasculares. Esto puede explicar parcialmente la duración del síndrome de abstinencia a metadona.

Este agonista opiáceo sufre una intensa metabolización hepática, responsable de su inactivación; por ello, una insuficiencia hepática, aumentaría los niveles libres del opiáceo. Sigue varias rutas metabólicas superpuestas, entre ellas destaca la N-desmetilación y formación de compuestos cíclicos. Estudios recientes (1) han señalado al citocromo P450 3A4 como sitio primario de desmetilación de la metadona y otros opiáceos en el hepatocito humano, habiéndose descrito la posibilidad de inhibición de esta isoenzima por la propia metadona (2). La metadona puede, a su vez, afectar al metabolismo de otros fármacos por inhibición del citocromo P450 2D6 (3), sistema de biotransformación utilizado por numerosas sustancias, caso de algunos opiáceos (codeína, dextrometorfano), antipsicóticos y antidepresivos. Los metabolitos inactivos de la metadona, junto con pequeñas cantidades sin modificar de la misma, se eliminan por orina. Un $20 \%$ de su eliminación es renal por lo que es recomendable modificar la dosificación en enfermos con disminución de la función renal. La acidificación de la orina aumenta la eliminación de metadona.
Su vida media es prolongada (15-40 h.), por lo cual puede ser administrada 2-3 veces a la semana en tratamientos sustitutivos. Esta característica, junto con el lento descenso de sus concentraciones plasmáticas (por liberación desde los tejidos extravasculares) es aprovechada en los programas de desintoxicación (1012 días) al tolerarse mejor la abstinencia por la menor brusquedad de los signos de deprivación.

\section{INTERACCIONES ENTRE METADONA Y FÁRMA- COS ANTIINFECCIOSOS}

Previamente hemos señalado la posible polimedicación en los sujetos incluidos en programas de mantenimiento con metadona (PMM) como consecuencia del tratamiento de enfermedades coexistentes. Entre ellas, destaca la posibilidad de adquisición de infecciones, fundamentalmente, infección por $\mathrm{VIH}$, dada la frecuente asociación de conductas de riesgo en el toxicómano. En efecto, un porcentaje no desestimable de los pacientes en tratamiento con metadona son portadores de VIH, una buena parte de los cuales desarrollará el síndrome de inmunodeficiencia. La terapéutica farmacológica en estos enfermos es complicada debido a causas muy diversas entre las que destaca el uso de distintos antiinfecciosos, sea en el tratamiento de la infección por VIH (antirretrovirales) o de las infecciones asociadas propias del síndrome de inmunodeficiencia (antibióticos, tuberculostáticos, antifúngicos,...). A menudo es necesario emplear en estas situaciones un aboradaje agresivo que supone la utilización frecuentemente conjunta de antibióticos y quimioterápicos con un serio perfil tóxico. Las interacciones de metadona con distintos fármacos antiinfecciosos pueden afectar a la eficacia del fármaco, su toxicidad y al cumplimiento de la pauta terapéutica. En la tabla II se recoge un resumen de las interacciones mejor documentales.

\section{FÁRMACOS ANTIRRETROVIRALES}

En el tratamiento farmacológico de la infección por VIH se sigue un abordaje múltiple con el fin de dificultar el fracaso por aparición de resistencias en el virus, disminuir la carga viral, la incidencia de infecciones oportunistas y, en consecuencia, aumentar la supervivencia y la calidad de vida del paciente. La combinación de fármacos antirretrovirales se ha convertido en una pauta estándar de tratamiento aceptada internacionalmente. Sin embargo, no está exenta de ciertos riesgos derivados de la toxicidad individual de estos fármacos y de las posibles interacciones. A ella hay que sumar el uso simultáneo de otros medicamentos 
Tabla II. Interacciones entre metadona y fármacos antiinfecciosos

\begin{tabular}{|c|c|c|}
\hline Fármaco & Consecuencia farmacocinética & Consecuencia clínica \\
\hline $\begin{array}{l}\text { Inhibidores de la proteasa } \\
\text { Eritromicina } \\
\text { Ketoconazol } \\
\text { Fluconazol }\end{array}$ & $\begin{array}{l}\uparrow \text { Niveles plasmáticos de } \\
\text { metadona }\end{array}$ & $\begin{array}{l}\text { Posibilidad } \uparrow \text { efectos opiáceos } \\
\text { (sedación, depresión respiratoria) }\end{array}$ \\
\hline $\begin{array}{l}\text { Rifampicina } \\
\text { Zidovudina? }\end{array}$ & $\downarrow$ Niveles plasmáticos de metadona & Síndrome de abstinencia \\
\hline Azidotimidina & Metadona aumenta sus niveles & $\begin{array}{l}>\text { Posibilidad de efectos } \\
\text { tóxicos dosis-dependientes }\end{array}$ \\
\hline
\end{tabular}

en el tratamiento de las infecciones asociadas, cuyos efectos secundarios pueden ser marcados. De hecho, los pacientes con SIDA han sido señalados como un grupo con especial incidencia y severidad de efectos adversos medicamentosos en comparación con el resto de la población $(4,5,6)$. En esta mayor susceptibilidad puede influir no sólo la importante polimedicación sino otros hechos como el trastorno imunológico subyacente o las lesiones multiorgánicas coexistentes que comprometen en muchos casos a órganos vitales y "detoxificadores". En definitiva, todo ello puede conducir a una respuesta distinta del organismo frente a la terapéutica farmacológica. Muestra de la complicada "situación farmacológica" de estos pacientes son los datos que calculan un amedia de 5.6 fármacos recibidos por paciente ambulatorio con $\mathrm{VIH}$ (7) y 9 en el hospitalizado (5), existiendo una relación entre incidencia de efectos secundarios y número de fármacos administrados y duración del ingreso hospitalario (5).

Los fármacos antirretrovirales disponibles en la actualidad utilizan dos mecanismo básicos de acción: 1) inhibición de la transcriptasa inversa, o retrotranscriptasa, responsable de la traducción del ARN viral al ADN eucariota, y 2) inhibición de la proteasa, enzima viral que actua en la fase final del ciclo evolutivo del virus, fundamental para la generación de nuevas partículas víricas infectantes. De esta forma, es posible actuar sobre las dos enzimas más importantes en el ciclo vital del VIH. El primer fármaco disponible y perteneciente al primer grupo fue la zidovudina; hoy existen varios fármacos con idéntico mecanismo de acción, unos cíclicos o análogos de los nucleósidos como ella (estavudina, lamivudina, didanosina, zalcitabina, abacavir -no comercializado en España-) y otros o no cíclicos, es decir, sin relación estructural con los nucleósidos (nevirapina, delavirdina). Algunos de estos fármacos muestran de forma aislada altos porcentajes de reacciones adversas graves, que se ven potencialmente agravadas por la coutilización de otras sustancias.
Los efectos de la zidovudina o azidotimidina (AZT) sobre la metadona son controvertidos, pues mientras unos autores señalan una disminución del efecto del opiáceo (8), otros no han podido constatar cambios en la farmacocinética de la metadona ni síntomas de abstinencia en pacientes con VIH en tratamiento con zidovudina (9). Sin embargo, la interacción potencial entre estos dos fármacos parece haberse visto confirmada pero en sentido contrario, es decir, los niveles plasmáticos del antirretroviral se ven afectados como consecuencia de la administración de metadona, que interfiere con la vía metabólica principal del antiviral. Así, en clínica se ha advertido un incremento en los niveles plasmáticos de zidovudina, aparentemente debido a una disminuciónd de su glucuronoconjugación hepática $(9,10,11)$, vía primaria de metabolización del AZT. Así, se observa una disminución paralela del metabolito glucurónido inactivo (GAZT) como consecuencia de la inhibición concentración-dependiente de la enzina implicada en esta reacción según se ha confirmado in vitro (12). En voluntarios sanos (13) se ha apreciado que tanto el tratamiento agudo como crónico con metadona aumentan los niveles bajo la curva de zidovudina administrada por vía oral o intravenosa como consecuencia de una disminución de su aclaramiento, mientras que los niveles de metadona permanecen en el rango terapéutico. Sin embargo, la inhibición de la glucuronidación de zidovudina por metadona debe tener una menor significación clínica que la atribuida a otros fármacos (fluconazol, por ejemplo) (12). En efecto, las concentraciones de metadona necesarias in vitro para alcanzar una inhibición significativa (50\%) de la formación de GAZT son muy superiores a las concentraciones plasmáticas que se alcanzan habitualmente en clínica (entrarían dentro del rango tóxico). Es posible que en las modificaciones plasmáticas comentadas también participe una disminución de la eleminación renal del antivírico. 
En definitiva, la afectación de los niveles de AZT por la administración simultánea de metadona puede revestir especial gravedad en relación con la toxicidad del antirretroviral, con una ventana terapéutica estrecha. La peligrosidad de esta interacción radica en la relación directa que la frecuencia y severidad de la supresión medular, su efecto indeseable más temido, guarda con las concentraciones plasmáticas de AZT. De forma que los pacientes en PMM que reciben dosis estándar de AZT están más expuestos a la misma y pueden ver aumentado el riesgo de reacciones adversas tóxicas. No obstante, la importancia de tal influencia farmacocinética parece limitada, no siendo necesario suspender el tratamiento antirretroviral. Se recomienda una especial vigilancia ante una posible infradosificación de metadona y/o toxicidad de zidovudina y, posiblemente, una reducción de la dosis de zidovudina.

Los inhibidores no nucleósidos de la transcriptasa inversa, de más reciente aparición, tienen una actividad antirretroviral inferior a los inhibidores de la proteasa, a los cuales se suponen una alternativa para casos en que éstos no pueden utilizarse (por ineficacia o intolerancia) o estadíos poco evolucionados de la enfermedad (14). Es pronto para conocer con certeza todo su perfil de interacciones, pero teniendo en cuenta su estrecha relación con las isoformas del sistema del citocromo P450, a través del cual se metabolizan, son esperables. Nevirapina y efavirenz -este último en desarrollo- inducen al sistema P450 y pueden disminuir las concentraciones plasmáticas de otros fármacos, incluidos los inhibidores de la proteasa. Delavirdina, aún no comercializada en nuestro país, es, al contrario, inhibidor de este sistema y puede aumentar las concentraciones de otras sustancias.

Los inhibidores de las proteasas son fármacos introducidos recientemente (en 1996) en el protocolo de tratamiento de la infección del VIH y, en todo caso, se utilizan siempre en combinación con inhibidores de la transcriptasa inversa. Estos fármacos (ritonavir, saquinavir, indinavir, nelfinavir, amprenavir -en fase de precomercialización-,...) son potentes inhibidores de los sistemas de citocromos hepáticos, responsables de la metabolización de muchos fármacos en el hombre. Concretamente, todos ellos inhiben a la isoenzima 3A4, implicada en el metabolismo de más del $50 \%$ de los fármacos, pudiendo por ello dar lugar a interacciones farmacocinéticas notables con aumento de los niveles plasmáticos y, por tanto, efectivos y/o tóxicos de otros fármacos. Más aún, estos antirretrovirales interactúan entre sí, afectando a la metabolización de los demás miembros del grupo e incluso pueden ser autoinductores enzimáticos. Saquinavir presenta la menor potencia inhibitoria -de hecho, es el de menor número de interacciones descritas- y ritonavir es el más potente; entre ambos se sitúan amprenavir, nalfinavir e indinavir (15). Consecuente- mente, dentro de este grupo farmacológico, ritonavir es el que presenta interacciones con mayor número de medicamentos a resultas de la inhibición del citocromo P450 3A4 (CYP 3A4). Esta isoforma del citocromo P450 es utilizada por la metadona para su metabolización (también buprenorfina o fentanilo) (1). De hecho, estudios in vitro han desvelado que ritonavir es el inhibidor de la proteasa que inhibe con mayor potencia el metabolismo de la metadona, seguido de indinavir y saquinavir (16). Por este motivo, se apuntó, de forma teórica, la necesidad de una reducción en la dosificación de metadona en caso de tratamiento simultáneo con inhibidores de la proteasa. De esta manera se evitaría el aumento de los efectos opiáceos tóxicos, particularmente la sedación extrema y/o depresión respiratoria derivadas de la depresión neurológica central.

Sin embargo, ninguno de estos fármacos presenta un perfil sencillo de efectos enzimáticos y las consecuencias clínicas de las interacciones metabólicas son difícilmente previsibles. Así, indinavir y saquinavir inhiben también la N-desmetilación del opiácoe (16). Por otro lado, ritonavir (el más estudiado) tiene acciones in vitro aún más complejas pues, además de inhibir potentemente a la isoenzima CYP $3 \mathrm{~A} 4$, también inhibe, aunque en menor grado, a CYP 2D6 e induce a diversas enzimas metabólicas (1A4, glucuronil-transferasa y probablemente $2 \mathrm{C} 9$ y $2 \mathrm{C} 19)$. Por todo ello, resulta complicado predecir las consecuencias finales originadas sobre el metabolismo de fármacos que siguen varias vías metabólicas como consecuencia de la presencia simultánea de ritonavir en el organismo. Posiblemente por esta razón, a pesar de prever un aumento de las concentraciones plasmáticas de metadona en presencia de ritonavir por la potente inhibición del citocromo P450, se ha descrito una disminución leve de las mismas, en las cuales se supone una participación de la inducción del CYP 2C9 (17).

En resumen, las constantes y complicadas interacciones a nivel farmacocinético obligan a una especial vigilancia y cautela cuando se coadministren inhibidores de la proteasa del HIV-1 y metadona. Posiblemente la limitada experiencia con estos fármacos (debido a lo relativamente reciente de su empleo extensivo), impide tener datos suficientes sobre la importancia clínica real de sus interacciones con metadona, pero sin duda en un corto lapso de tiempo iran conociéndose.

\section{OTROS ANTIINFECCIOSOS}

En la actualidad, la tuberculosis representa un problema de salud pública en todo el mundo. En España, las tasas de tuberculosis se mantienen en torno a los 40 casos por 100.000 habitantes y año (18). Dentro de este contexto epidemiológico de alta prevalencia e 
incidencia de tuberculosis, la infección por VIH y el uso de drogas por vía parenteral u otras, constituyen dos factores de riesgo de desarrollo de la infección tuberculosa. Por este motivo, la población incluida en PMM presenta una probabilidad relativamente alta de padecer infección por Mycobacterium tuberculosis (u otras micobacterias). Además, la terapéutica antituberculosa presenta características peculiares que podemos resumir en la necesidad de un tratamiento plurifarmacológico durante un período de tiempo relativamente prolongado (aunque hoy se tiende a utilizar pautas de corta duración) y de una buena adhesión al protocolo para una correcta eficacia. Por otro lado, los fármacos antituberculosos son un grupo terapéutico de características especiales determinadas por la alta incidencia de efectos adversos, algunos de los cuales puede conducir al abandono del tratamiento. De entre ellos, detacamos por su trascendencia en el tema que nos ocupa, la hepatotoxicidad por isoniazida, rifampicina o pirazinamida.

Entre los tuberculostáticos mayores, la rifampicina es responsable de una importante y conocida interacción farmacológica con metadona, de considerable trascendencia clínica por su alta incidencia. En efecto, la rifampicina disminuye de forma marcada los niveles plasmáticos de metadona, pudiendo necesitarse un importante aumento de las dosis de ésta para evitar la aparición de síntomas de abstinencia, cuya presentación puede ser muy dilatada $(8,19,20,21)$. Esta interacción se debe al potente efecto inductor enzimático de la rifampicina, que afecta a las enzimas hepáticas participantes en el metabolismo de la metadona. Por ello, la administración conjunta de rifampicina disminuye los niveles plasmáticos de metadona y aumenta la excreción de su metabolito mayor $(19,22)$. El porcentaje de pacientes que presentan este problema varía según las series pero es, en todos los casos, notable. Puesto que el tratamiento antituberculoso tiene una larga duración y requiere un buen cumplimiento para su éxito, se recomienda no suspenderlo sino aumentar las dosis de metadona durante el tratamiento tuberculostático.

La eritromicina, un macrólido empleado, por ejemplo, en el tratamiento de neumonías atípicas, aumenta las concentraciones plasmáticas de metadona por inhibición de los sistemas enzimáticos hepáti$\cos (19)$.

También las infecciones por hongos, en muchos casos sistémicas, se presentan en los sujetos con inmunodepresión, requiriendo en ocasiones un ataque bastante agresivo para su erradicación. De entre los fármacos antimicóticos, se ha descrito un aumento de las concentraciones plasmáticas de metadona por ketoconazol, originado por la inhibición del metabolismo hepático del opiáceo (19). De hecho, es capaz de disminuir la $\mathrm{N}$-desmetilación de metadona a menos del $40 \%$, a diferencia de otros inhibidores del citocro- mo P450 (1) y esta inhibición puede afectar también a otras sustancias utilizadas en el tratamiento de los adictos a opiáceos como el levo-alfa-acetilmetadol (LAAM). También fluconazol, otro derivado imidazólico, afecta a la disponibilidad de metadona, pues tras tratamiento continuado con este antifúngico se ha observado un aumento de los niveles de metadona plasmática, incluido el pico máximo (27\%), en sujetos sanos. En este efecto se ha implicado una disminución de la eliminación de la misma del organismo (23). Sin embargo, a pesar del aumento de las concentraciones del agonista opiáceo, los pacientes sometidos a tratamiento con fluconazol no exhibieron, en el estudio referido, síntomas o signos de sobredosis opiácea.

\section{INTERACCIONES DE METADONA CON OTRAS SUSTANCIAS DE ABUSO}

En las personas con adicción a opiáceos es muy frecuente la ingesta de otras sustancias con capacidad adictiva. Entre ellas destacan algunos psicofármacos como las benzodiacepinas que pueden aumentar los efectos depresores de metadona (revisión en Moreno-Brea y cols. (24). Con frecuencia utilizan, además, otros tóxicos de forma simultánea e independientes del control médico. Es muy importante conocer las posibles interferencias que estos pueden tener con la metadona, sobre todo por la ausencia de supervisión y desconocimiento por parte de los terapeutas que controlan los PMM.

\section{ALCOHOL}

El abuso del alcohol es un problema complejo siempre, pero aún más cuando se presenta en sujetos con historia de abuso de otras sustancias, como es el caso de los individuos incluidos en PMM. Este sector de población tiende a consumir todo tipo de drogas psicoactivas, con el consecuente riesgo de sobredosis por potenciación de sus efectos $y / 0$ aumento de sus niveles. Precisamente es el alcohol junto a las benzodiacepinas, una de las combinaciones encontradas con mayor frecuencia en las muertes relacionadas con la metadona $(25,26)$. La trascendencia de esta combinación queda subrayada por el hallazgo de menores concentraciones plasmáticas de metadona con defunciones relacionadas con alcohol que en aquellas en que éste no estuvo presente, a pesar de que las primeras sólo constituyeron un 30\% de los casos en este estudio (27).

La adicción al alcohol en pacientes a su vez adictos a opiáceos, se ha relacionado con una mayor prevalencia de problemas psiquiátricos (28). En cuanto al 
consumo, existen discrepancias entre diversos autores sobre si aumenta o disminuye después del ingreso del paciente en un $\operatorname{PMM}(29,30)$, siendo este un tema de debate. Parece ser que, aunque el consumo de alcohol es elevado en los pacientes en PMM, la mayoría ya consumía alcohol antes de entrar en el programa. En algunos estudios, se ha observado que el aumento de la tasa de alcohólicos puede deberse simplemente a un progresivo descubrimiento de los hábitos del paciente por parte del personal médico en los casos de larga evolución, consecuente al mayor trato (31); sin embargo tomar esta afirmación como definitiva puede ser arriesgado. Por otro lado, Chatham y cols. (32) descubren, inesperadamente, que los pacientes dependientes al alcohol permanecen más tiempo en los PMM que los bebedores no dependientes, y lo justifican por el hecho de que aquellos suelen haber tenido experiencias anteriores con grupos de apoyo, suelen ser más conscientes de su problema y están más dispuestos a remediarlo. El estudio de Herd (33) encuentra que los individuos encarcelados con historial de abuso de sustancias y en programas exhibían los niveles más altos de consumo de alcohol, los integrados en comunidades terapéuticas tasas intermedias, y los ambulatorios en PMM, las tasas más bajas de consumo alcohólico. Parece, pues, que el mejor predictor de un consumo elevado de alcohol, así como de las combinaciones alcohol-drogas, son el contexto social y de bebida del paciente.

La sensación de bienestar que produce el etanol es, en principio, diferentes de la euforia inducida por los opioides. Sin embargo, puede existir una conexión entre estas sensaciones que implican a sistemas de refuerzo y recompensa. Aunque no existen datos fiables, una proporción considerable de adictos manifiesta buscar el consumo de alcohol un refuerzo del efecto de la metadona. No obstante, algunos estudios han mostrado un patrón de preferencia bimodal por el alcohol en los pacientes en PMM. Así, para algunos tiene un efecto psicotrópico agradable (puntuación 2, en una escala de $-5=$ muy desagradable $a+5=$ muy placentero) y para otros claramente desagradable (puntuación -3, en la misma escala) (34).
Una cuestión diferente a tener en cuenta depende del efecto del etanol sobre las membranas lipídicas, donde se ubican los receptores para neurotransmisores, como los opioides. Algunos estudios realizados en animales de experimentación, revelan que el alcohol puede producir alteraciones en los niveles de péptidos opioides endógenos y en su afinidad por algunos receptores opioides. Estudios in vitro han mostrado una interacción a nivel membranario entre opioides y alcohol que va más allá del posible efecto sobre los receptores opioides (35)

La interacción entre el alcohol y metadona a nivel farmacocinético es bien conocida y se produce por competición a nivel metabólico (36). Ambos fármacos son sustratos de las enzimas oxidativas del sistema citocromo P450. El alcohol es un inductor de estas enzimas, mientras que la metadona las induce débilmente, acelerándose su metabolismo tras tratamiento crónico. Así, cuando se combina con alcohol, la metadona se metaboliza más lentamente (por competencia por las enzimas); por ello, ingestas agudas de grandes dosis de alcohol junto a metadona pueden provocar una sedación intensa y depresión respiratoria. Sin embargo, en administración crónica, el alcohol puede conducir a una metabolización más rápida (por inducción enzimática) y niveles tisulares más bajos de metadona, provocando una aparición más precoz del síndrome de abstinencia y surgiendo la necesidad de aumentar la dosis del opiáceo (tabla III). A pesar de ello, estudios metabólicos demuestran que dosis moderadas de alcohol en pacientes, relativamente sanos, establizados en PMM no alteran los niveles del sustituto y que los niveles de etanol no varían si se suprime la dosis de metadona. Así, parece ser que la metadona crónica per se no es capaz de provocar alteraciones tales en el metabolismo del alcohol que induzcan cambios en los patrones de consumo de estos sujetos (31).

La inhibición de la secreción pancreática inducida por metadona puede verse aumentada por el alcohol, siendo este efecto de tipo aditivo, sin potenciación (37). La coincidencia de estos factores puede ser

Tabla III. Interacciones entre metadona y alcohol

\begin{tabular}{lccc}
\hline Consumo de alcohol & Mecanismo & $\begin{array}{c}\text { Consecuencia } \\
\text { farmacocinética }\end{array}$ & $\begin{array}{c}\text { Consecuencia } \\
\text { clínica }\end{array}$ \\
\hline $\begin{array}{l}\text { Ingesta de grandes } \\
\text { dosis agudas }\end{array}$ & $\begin{array}{c}\text { Competencia } \\
\text { enzimática }\end{array}$ & $\begin{array}{c}\text { Niveles plasmáticos de } \\
\text { metadona }\end{array}$ & $\begin{array}{c}\text { Sedación } \\
\text { Depresión } \\
\text { respiratoria }\end{array}$ \\
\hline Ingesta crónica & Inducción & $\downarrow$ Niveles plasmáticos de & Síndrome de \\
& enzimática & metadona & abstinencia
\end{tabular}


importante en bebedores crónicos, en los cuales es más frecuente la patología pancreática.

A pesar de todos estos datos, no llega a estar definitivamente claro cuál es realmente la importancia de la sinteracciones que se producen como consecuencia del abuso de alcohol por personas dependientes de opiáceos. La producción de sustancias de tipo opioide por parte del etanol, la mediación de algunos efectos del alcohol por opioides endógenos y las alteraciones de la unión de los opioides a sus receptores son algunas de las vías comunes (para revisión, Ulm. y cols., 1995 (38). Es necesario estudiar el contexto social del paciente y hacer una distinción entre bebedores no dependientes y pacientes adictos al alcohol, ello podría ayudarnos a establecer el diferente pronóstico en la evolución del tratamiento y las posibles interacciones. Finalmente, aunque el uso moderado de etanol en pacientes en PMM no tiene por qué verse afectado por el uso del opiáceo, no hay que olvidar la potencial peligrosidad del uso incontrolado de alcohol en estos pacientes, ya esta combinación está presente en un gran porcentaje de las muertes relacionadas con metadona.

\section{COCAÍNA}

El consumo de cocaína por pacientes incluidos en PMM es un hecho repetidamente constatado en muy diversos estudios epidemiológicos realizados en diferentes países $(39,40)$ incluido el nuestro $(41,42)$. Sin embargo, no existe uniformidad en los resultados referentes a la tendencia de dicho consumo bajo influencia del tratamiento con metadona, se observa que en los últimos años el uso de cocaína se ha multiplicado 1.5 veces entre los pacientes en PMM (43). Por contra, otros autores habían encontrado previamente una disminución del uso de cocaína (44). Así pues, las conclusiones a propósito de los efectos del tratamiento con metadona sobre la adición a cocaína son muy variables; se ha señalado que el efecto depende del tipo de cocaína y administración empleadas (45), oscilando los resultados desde una disminución del uso de cocaína (46) a la inexistencia de cambios observables en su patrón de consumo (47), pasando por un inicio de la adición o aumento del consumo tras comenzar el programa con el agonista opiáceo (48). Un estudio experimental clínico (49) ha constatado que la administración de cocaína en sujetos en PMM, ocasiona un aumento de los efectos subjetivos de la cocaína respecto a la dosis de metadona o al calendario de administración, sugiriendo que si existe alguna mejor en el abuso de cocaína en sujetos incluidos en PMM, no se debe al bloqueo o anulación de los efectos subjetivos de la cocaína.

Dado que la cocaína actúa primariamente sobre la neurotransmisión catecolaminérgica, la interacción de ésta con los sistemas opioides centrales pudiera, teó- ricamente, ser responsable de interacciones entre cocaína y metadona. Diversos datos derivados de trabajos experimentales permiten suponer ciertas relaciones farmacológicas entre ambas que pudieran subyacer en el origen de posibles interacciones. Así, la cocaína potencia la analgesia de diferentes agonistas opiáceos con una aparente implicación de la noradrenalina en este efecto (50). También se ha observado que el pretratamiento con metadona u otros opiáceos potencia el efecto discriminativo de la cocaína y aumenta sus propiedades reforzadoras de la misma en place preference (51), propiedades que son bloqueadas por antagonistas opiáceos o agonistas kappa. A partir de estos resultados experimentales, algunos autores han sugerido la utilidad de los antagonistas opiáceos pudieran en el tratamiento de la adicción a cocaína e incluso una explicación posible a las altas tasas de uso de cocaína en adictos a heroína tratados con metadona. Por último, se ha encontrado una disminución del número de receptores $\beta$-adrenérgica y $\delta$-opioidérgica en placentas procedentes de gestaciones en consumidoras de cocaína durante el embarazo (cambios que pudieran subyacer en algunos de los frecuentes problemas que surgen en su curso en estas mujeres) (52). Se desconoce si esta downregulation de los receptores mencionados ocurre a otros niveles y si tiene significación clínica o participa en algún efecto, sobre todo opiode, de la cocaína. Resulta muy aventurado pronunciarse a este respecto sin más evidencias científicas. La complejidad de las relaciones existentes entre los sistemas de neurotransmisión implicados en las conductas adictivas no permite una explicación.

Apenas se encuentran, por otro lado, interacciones clínicas confirmadas documentalmente entre metadona y cocaína. En pacientes mantenidos con metadona y dependientes de cocaína, se ha observado la presentación de síntomas de abstinencia a cocaína moderados y de corta duración (53); de hecho, el síndrome de abstinencia desencadenado por naloxona en estos sujetos es de intensidad inversamente proporcional a la dependencia a cocaína y a otras drogas (54). Igualmente, se ha indicado que el aumento de la incidencia de ataques de pánico en los pacientes con metadona se relaciona con el consumo simultáneo de cocaína (55). Por otra parte, el uso de heroína y metadona se asocia con edema pulmonar y aunque su asociación con cocaína es un hallazgo postmortem común, son pocos los casos descritos diagnosticado clínicamente tras el uso de cocaína (56).

\section{CÁNNABIS Y ÉXTASIS.}

Otras sustancias de abuso frecuentemente utilizadas por los pacientes en programa de deshabituación/mantenimiento con opiáceos, son cánnabis o 
anfetaminas $(57,58)$, cuyo uso parece aumentar en estos pacientes tras la introducción en programas de metadona (59). En nuestro país, el grupo de San (42) ha señalado el alto consumo de estas sustancias junto con cocaína. Las consecuencias de tal utilización conjunta son difíciles de prever, así como prácticamente inexistentes las investigaciones al respecto, proporcionando tan sólo datos aislados. Se ha estudiado, por ejemplo, si determinados parámetros psicológicos (escala del sentido de la coherencia) variaban en usuarios de cánnabis, observándose que aquellos en tratamiento con metadona presentaban puntuaciones por debajo de las normales a diferencia de quienes sólo usaban cánnabis (60). Desconocemos, sin embargo, la trascendencia práctica de este hallazgo.

En cuanto a las anfetaminas y sus derivados, se sabe que dosis bajas de anfetaminas aumentan sustancialmente los efectos analgésicos y euforizantes de la morfina y pueden disminuir los efectos adversos sedativos de ésta. Con estos datos se ha intentado dar una justificación teórica a la utilización de anfetaminas en la comunidad dependiente de opiáceos. Las "drogas de diseño", derivados anfetamínicos, como el MDMA ("éxtasis"), han cobrado gran importancia como sustancia ilegal o droga de abuso en nuestro país (61), pero han sido menos estudiadas en relación con la metadona. Se ha observado que un porcentaje considerable de sujetos consumidores de cocaína y/o heroína usaban en además este tipo de sustancias (61). En cuanto a las consecuencias de su consumo, existen numerosas comunicaciones acerca de los importantes efectos tóxicos del MDMA y sus derivados, que pueden producir reacciones adversas agudas intensas, incluso mortales (62). Se sabe que el "éxtasis" y otros derivados anfetamínicos pueden originar cuadros neurológicos mixtos entre el síndrome neuroléptico maligno y el síndrome serotoninérgico, cuadros psicóticos (63), alteraciones renales (64) y hepáticas $(65,66)$. No obstante, hasta el presente, no se conoce la influencia que en tales u otros efectos pudiera tener el consumo simultáneo de metadona.

\section{NICOTINA}

Los agonistas opiáceos pueden aumentar el número de cigarrillos consumidos (67) y viceversa, tras la ingestión de nicotina (cigarrillo o chicle) se ha observado un aumento del consumo de metadona (68). Sin embargo, las relaciones entre nicotina y sistema opioide son muy complejas y permanecen sin desentrañar convenientemente, existiendo muchas contradicciones en el estado de conocimiento actual. Por ejemplo, algunos efectos de la nicotina son bifásicos, pro-opioides (agonistas) a dosis altas y antagonistas a dosis bajas, la contribución de los receptores opiáceos varía según las dosis de nicotina estudiadas, etc. Sin duda, en fin, la neurotransmisión opioide participa a través de los sistemas de recompensa centrales, en la adquisición del hábito tabáquico e incluso puede reforzarlo primariamente sobre todo en presencia de estrés (67). A pesar de carecer de resultados definitivos, es evidente la existencia de influencias recíprocas entre el consumo de metadona y de nicotina. Por ello, merece la pena llamar la atención sobre un hecho (fumar) que suele pasar desapercibido ante la trascendencia de las consecuencias de interacciones de metadona con otras sustancias potencialmente más peligrosas. Posiblemente también la mayor aceptación social del hábito tabáquico contribuye a un menor control o información a propósito del mismo en los pacientes que toman metadona.

En definitiva, los datos existentes alertan sobre la alta probabilidad de interacciones farmacológicas en los sujetos en tratamiento de mantenimiento con metadona. Por este motivo resulta conveniente realizar un especial seguimiento de estos pacientes y su historia fármaco-toxicológica. Ello nos permitirá conocer la verdadera incidencia y trascendencia clínica de tales interacciones.

\section{BIBLIOGRAFÍA}

(1) Moody DE, Alburges ME, Parker RJ, Collins JM, Strong $\mathrm{JM}$. The involvement of cytochrome P450 3A4 in the Ndemethylation of L-alpha-acetylmethadol (LAAM), norLAAM, and methadone. Drug Metab Dispos 1997; 25:1347-53.

(2) Iribarne C, Dreano Y, Bardou LG, Menez JF, Berthou F. Interaction of methadone with substrates of human hepatic cytochrome P4503A4. Toxicology 1997; 117:13-23.

(3) Wu D, Otton SV, Sproule BA, et al. Inhibition of human cytochrome P4502D6 (CYP2D6) by methadone. Br J Clin Pharmacol 1993;35:30-4.

(4) Lee BL, Safrin S. Interactions and toxicities of drugs used in patients with AIDS. Clin Infect Dis 1992;14:773-9.

(5) Harb GE, Alldredge BK, Coleman R, Jacobson MA. Pharmacoepidemiologyy of adverse drug reations in hospitalized patients with human immunodeficiency virus disease. J Acquir Immune Defic Synd 1993;6:919-26.

(6) Hughes WT, LaFon SW, Scott JD, Masur H. Adverse events associated with trimethoprim-sulphametoxazole and atovaquone during treatment of AIDS-related pneumocystis carinii pneumonia. J Infect Dis 1995; 171:1295-301.

(7) Greenblatt RM, Hollander H, McMaster JR, Henke CJ. Polypharmacy among patientes attending on AIDS clinic:utilization of prescribed, unorthodox, and investigational treatments. J Acquir Immune Defic Synd 1991;4:136-43. 
(8) Brockmeyer NH, Mertins L, Goos M. Pharmacokinetic interaction of antimicrobial agents with levomethadone in drug-addicted AIDS patients. Klin Wschr 1991;69:168.

(9) Schwartz EL, Brechbuhl AB, Kahl P, Miller MA, Selwyn $\mathrm{PA}$, Friedland $\mathrm{GH}$. Pharmacokinetic interactions of zidovudine and methadone on intravenous drug-using patients with HIV infection. J Acquier Immune Defic Synd 1992;5:619-26.

(10) Bruger DM, Meenhorst PL, Ten-Napel CH, et al. Pharmacokinetic variability of zidovudine in HIV-infected individuals: subgroup analysis and drug interactions. AIDS 1994;8(12):1683-9.

(11) Jatlow P, McCance EF, Rainey PM, Trapnell CB, Friedland G. Methadone increases zidovudine exposure in HIVinfected injection drug users. j1996. Abstracts of the 3rd Conference on Retroviruses and Opportunistic Infections, p. 129. Washington D.C.

(12) Trapnell CB, Klecker RW, Jamis-Dow C, Collins JM. Glucuronidation of 3'-azido-3'deoxythymidine (zidovudine) by human liver microsomes: relevance to clinical pharmacokinetic interactions with atovaquone, fluconazole, methadone and valproic acid. Antimicrob Agents Chemother 1998;42(7):1592-6.

(13) McCance Katz EF, Rainey PM, Jatlow P, Friedland G. Methadone effects on zidovudine disposition (AIDS Clinical Trials Group 262). J Acquir Immune Defic Syndr Hum Retrovirol 1998;18(5):435-43.

(14) Hirsch MS. Current antiretrovirals -a review. Antiviral Ther 1997;2(Suppl 4):19-40.

(15) Decker CJ, Laitine LM, Bridson GW, Raybuck SA, Tung RD, Chaturvedi PR. Metabolism of amprenavir in liver microsomes: role of CYP3A4 inhibition for drug interactions. J Pharm Sci 1998;87(7):803-7.

(16) Iribarne C, Berthou F, Carlhant D, et al. Inhibition of methadone and buprenorphine $\mathrm{N}$-dealkylations by three HIV-1 protease inhibitors. Drug Metab Dispos 1998;26(3):257-60.

(17) Hsu A, Granneman GR, Bertz RJ. Ritonavir. Clinical pharmacokinetics and interactions with other anti-HIV agents. Clin Pharmacokinet 1998;35(4):275-91.

(18) Sauret J. Situación actual de la tuberculosis en España. Conferencia Internacional sobre Tuberculosis. Escuela Anzaluza de Salud Publica, Granada, 1996.

(19) Kreek MJ, Garfield JN, Gutjah CL, Giusti LM. Rifampicin-induced methadone withdrawal. N Engl J Med 1976;294:1104-6.

(20) Garfield JW, Kreek MJ, Giusti L. Rifampicin-methadone relationship. 1. The clinical effects of rifampicin-methadone interaction. Am Rev Respir Dis 1975;111:262.

(21) Bending MR, Skacel PO. Rifampicin and methadone withdrawal. Lancet 1997;i:1211.

(22) Kreek MJ, Garfield JW, Gutjah CL, Bowen D, Field F, Rotschild M. Rifampicin-methadone relationship.2. Rifampicin effects on plasma concentration, metabolism and excretion of methadone. Am Rev Respir Dis 1975;111:926-7.
(23) Cobb MN, Desai J, Brown LS, Zannikos PN, Rainey PM. The effecto of fluconazole on the clinical pharmacokinetics of methadone. Clin Pharmacol Ther 1998;63:655-62.

(24) Moreno Brea MR, Rojas Corrales O, Gibert-Rahola J, Micó Segura JA. Interacciones medicamentosas de metadona con psicofármacos. Actas Esp Psiquiatr 1999;27(2):103-10.

(25) Barrett DH, Luk AJ, Parrish RG, Jones TS. An investigation of medical examiner cases in which methadone was detected, Harris County, Texas, 1987-1992. J Forensic Sci 1996;41(3):442-8.

(26) Williamson PA, Foreman KJ, White JM, Anderson G. Methadone-related overdose deaths in South Australia, 1984-1994. Med J Aust 1997;166:302-5.

(27) Worm K, Steentoft A, Kringsholm B. Methadone and drug addicts. Int J Legal Med 1993;106:119-23.

(28) El-Bassel N, Schilling RF, Turnbull JE, Su KH. Correlates of alcohol use among methadone patients. Alcohol Clin Exp Res 1993;17(3):681-6.

(29) Barr HL, Cohen A. The problem of drinking drug addict. In: Gardner SE, ed. National Drug/Alcohol Colalborative Project: Issues in Multiple Substance Abuse, DHEV $n^{\circ}$ (ADM) 80-957. Washington: Government Printing Office, 1980:78-115.

(30) Stimmel B, Cohen M, Stuariano V. Is treatment for alcoholism effective in persons on methadone maintenance? Am J Psychiatry 1983;140:862-6.

(31) Cushman P. Alcohol and opioids: possible interactions of clinical importance. Adv Alcohol Subst Abuse 1987;6(3):33-46.

(32) Chatham LR, Rown-Szal GA, Joe GW, Brown BS, Simpson DD. Heavy drinking in a population of methadonemaintained clients. J Study Alcohol 1995;56(4):417-22.

(33) Herd D. Correlates of heay drinking and alcohol related problems among men and women in drug treatment programs. Drug Alcohol Depend 1993;32:25-53.

(34) Barnas C, Rossman M, Roessler H, Reimer Y, Fleischhacker WW. Benzodiacepines and other psychotropic drugs abused by patiens in a methadone maintenance program: familiarity and preference. J Clin Psychopharmacol 1992;12:397-402.

(35) Wu C, Henry JA. Interaction between ethanol and opioids in a protozoan assay. Hum Exp Toxicol 1994;13:145-8.

(36) Borowsky SA, Lieber CS. Interaction of methadone and ethanol metabolism. J Pharmacol Exp Ther 1979;207:123-31.

(37) Nagain-Domaine C, Tsocas A, Presset O, Roze C, Vaille C. Modulatin by alcohol and methadone of 2-deoxyglucose-stimulated pancreatic secretion in the rat. Fundam Clin Pharmacol 1996;10:538-46.

(38) Ulm RR, Volpicelli JR, Volpicelli LA. Opiates and alcohol self-administration in animals. J Clin Psychiatry 1995;56(Suppl. 7):5-14.

(39) Huber-Stemich F, Haas H. Prevention of HIV infection in the methadone program. A survey of a drop-in clinic in Zurich. Schweiz Rundsch Med Prax 1990;79:1017-21. 
(40) Black JL, Dolan MP, Penk WE, Robinowitz R, DeFord $H A$. The effect of increased cocaine use on drug treatment. Addict Behav 1987;12(3):289-92.

(41) Torrens M, San L, Peri JM, Olle JM. Cocaine abuse among heroin addicts in Spain. Drug Alcohol Depend 1991;27(1):29-34.

(42) San L, Torrens M, Castillo C, Porta M, de la Torre R. Consumption of buprenorphine and other drugs among heroin addicts under ambulatory treatment: results from cross-sectioned studies in 1988 and 1990. Addict Behav 1993;88(10):1341-9.

(43) Craddock SG, Rounds-Bryant JL, Flynn PM, Hubbard $\mathrm{RL}$. Characteristics and pretreatment behaviors of clients entering drug abuse treatment: 1969 to 1993. Am J Drug Alcohol Abuse 1997;23(1):43-59.

(44) Shaffer HJ, LaSalvia TA. Patterns of substance abuse among methadone maintenance patients. Indicators of outcome. J Subst Treat 1992;9(2):143-7.

(45) Grella CE, Anglin MD, Wugalter SE. Patterns and predictors of cocaine and crack use by cients in standard and enhanced methadone maintenance treatament. Am J Drug Alcohol Abuse 1997;23(1):15-42.

(46) Dunteman GH, Condelli WS, Fairbank JA. Predicting cocaine use among methadone patients: analysis of findings from a national study. Hosp Community Psyhiatry 1992;43(6):608-11.

(47) Kosten TR, Rounsaville BJ, Kleber HD. A 2.5 year followup of cocaine use among treated opioid addicts. Have our treatments helped? Arch Gen Psychiatry 1987;44(3):281-4.

(48) Chaisson RE, Bacchetti P, Osmond D, Brodie B, Sande MA, Moss AR. Cocaine use and HIV infection inf intravenous drug users in San Francisco. JAMA 1989;261(4):561-5.

(49) Foltin RW, Christiansen I, Levin FR, Fischman MW. Effects of single and multiple intravenous cocaine injections in humans maintained on methadone. J Pharmacol Exp Ther 1995;275:38-47.

(50) Misra AL, Pontani RB, Vadlamani NL. Stereospecific potentiation of opiate analgesia by cocaine: predominant role of noradrenaline. Pain 1987;28(1):129-38.

(51) Bilsky EJ, Montegut MJ, Delong CL, Reid LD. Opioidergic modulation of cocaine conditioned place preference. Life Sci 1992;50(14):PL85-90.

(52) Wang $\mathrm{CH}$, Schnoll SH. Prenatal cocaine use associated with down regulation of receptors in human placenta. Neurotoxicol Teratol 1987;9(4):301-4.

(53) Margolin A, Avants SK, Kosten TR. Abstinence symptomatology associated with cessation of chronic cocaine abuse among methadone-maintained patients. Am J Drug Alcohol Abuse 1996;22(3):377-88.

(54) Kosten TA, Jacobsen LK, Kosten TR. Severity of precipitated opiate withdrawal predicts drug dependence by DSM-III-R criteria. Am J Drug Alcohol Abuse 1989;15(3):237-50.

(55) Rosen MI, Kosten T. Cocaine-associated panic attacks in methadone-maintained patients. Am J Drug Alcohol Abuse 1992;18(1):57-62.

(56) Cucco RA, Yoo OH, Cregler L, Chang JC. Nonfatal pulmonary edema after "freebase" cocaine smoking. Am Rev Respir Dis 1987;136(1):179-81.

(57) Worm K, Steentoft A. Amphetamine abuse in Denmark. Ungeskr Laeger 1992; 154:2753-6.

(58) Olesen OV, Linnet K, Rosenberg R. Results of urinary control analyses of narcotic addicts on methadone therapy. An evaluation in the county of Arhus in 1993. Ugeskr Laeger 1995;157:6.

(59) Swensen G, llett KF, Dusci LJ, et al. Patterns of drug use by participants in the Western Australian methadone program, 1984-1991. Med J Aust 1993;159(6):373-6.

(60) Lundqvist T. Chronic cannabis use and the sense of coherence. Life Sci 1995;56(23-24):2145-50.

(61) De la Fuente de Hoz L, Rodríguez Arenas MA, Vicente Orta J, Sánchez Paya J, Barrio Anta G. Epidemiología del consumo de drogas de diseño en España. Med Clin (Barc) 1997;108:54-61.

(62) Green AR, Cross A, Goodwin GM. Review of the pharmacology and clinical pharmacology of 3,4-methylenedioxymethamphetamine (MDMA or "Ecstasy"). Psychopharmacoloby (Berl) 1995;119(3):247-60.

(63) McGuire PK, Cope H, Fahy TA. Diversity of psychopathology with use of 3,4-methylenedioxymethamphetamine ("Ectasy"). Br J Psychiatry 1994;165(3):391-5.

(64) Bakir AA, Dunea G. Drugs of abuse and renal disease. Curr Opin Nephrol Hypertens 1996;5(2):122-6.

(65) Dykuizen RS, Brunt PW, Atkinson P, Simpsom JG, Smith CC. Ecstasy induced hepatitis micmiking viral hepatitis. Gut 1995;36(6):939-41.

(66) Pascual Bartolomé S, Sarrión Martínez JV, García Herola A, Berenguer Lapuerta J. Hepatitis y éxtasis. Med Clin (Barc) 1997;108:279.

(67) Pomerleau F. Endogenous opioids and smoking: a review of progress and problems. Psychoneuroendocrinology 1998;23(2):115-30.

(68) Spiga R, Schmitz J, Day J. Effects of nicotine on methadone self-administration in humans. Drug Alcohol Depend 1998;50(2):157-65. 
